\title{
BEARING ANGLE MODEL FOR BOND OF REINFORCING BARS TO CONCRETE
}

\author{
Oan Chul Choi and Seung Yul Yang \\ Department of Architectural Engineering, Soong Sil University, Seoul, Korea \\ Department of Architectural Engineering, Soong Sil University, Seoul, Korea
}

\begin{abstract}
The rib geometry of deformed bars governs bond behavior and is instrumental in guaranteeing adequate bond resistance. The ribs of bars can split the concrete cover and shear off the concrete key by wedging action. This study is intended to explain the nature of the wedging action of ribbed bars as they interact with concrete during bond failure.

Analytical expressions to predict bond resistances for splitting and shearing are derived, in which the bearing angle is a key variable. As the bearing angle is decreased, the splitting bond resistance decreases while the shearing bond resistance increases. In the case of bars at a moderate level of confinement, the bearing angle is decreased to decrease the splitting resistance and to increase the shearing resistance, and then the concrete key is sheared off.

The bearing angle is determined so that the splitting resistance can be equal to the shearing resistance. The bearing angle model is proposed to better understand bond mechanisms between ribbed reinforcing bars and concrete.
\end{abstract}

\section{INTRODUCTION}

Modern deformed bar rib geometries date from the work of Clark in 1949. Since then, knowledge concerning the bond between concrete and ribbed deformed steel has considerably increased based on both experimental work and analytical studies. During the late 1950s and the 1960s, researchers observed two phenomena accompanied by the slip of ribbed bars: (1) concrete is split by the wedging action of the ribs and (2) concrete between the ribs is crushed (Rehm 1957, Lutz and Gergely 1967). Researchers observed that the ribs act as wedges and the concrete in front of the ribs crushes gradually, resulting in a pullout-type failure and found that the concrete in front of the ribs undergoes gradual crushing (Fig.1).

A number of researchers have derived analytical expressions for bond mechanisms in splitting failure (Tepfers 1979, Cairns 1979). Bond between steel bars and concrete has been idealized in finite element analyses. For the case of splitting failure, analytical studies of interfacial bond have been performed to predict the bond strength of ribbed reinforcing bars (Choi and Lee 2002), and in this paper, the role of the bearing angle on bond behavior is addressed.

The rib geometry of deformed bars governs bond behavior and is instrumental in guaranteeing adequate bond resistance. The influence of deformation pattern on bond performance has been studied and bond resistances have been observed to vary with the rib characteristics (Skorobogatov and Edwards 1979). Studies by Tholen and Darwin (1996) have demonstrated that bond strength increases with an increase in the relative rib area bars under high confinement, but under low confinement, bond strength is independent of deformation pattern. 
With this information as background, this study is intended to explain the nature of the wedging action of ribbed bars as they interact with concrete during bond failure. Analytical expressions to determine bond resistances for splitting and shearing failures are derived and used to predict bond strength. The roles of the bearing angle, which is the key variable in the expressions, are explored. The bearing angle model is proposed for analyzing the bond behavior of ribbed reinforcing bars to concrete and improving the understanding of bond mechanisms of ribbed reinforcing steel in concrete structures.

\section{BOND RESISTANCES IN SPLITTING AND SHEARING FAILURE}

\section{Bond resistance in splitting failure}

Wedging action by the rigid steel rib of deformed bars makes it possible to resolve bond forces into normal stress $\sigma_{n}$ and tangential shear stress $\tau$, The resultant of normal components along the bar is what places the surrounding concrete in tension. When a reinforcing bar in tension $\mathrm{P}$, concrete under the bearing side of a rib is placed in a state of tri-axial compression, with the major principal stress, the bearing stress, $\sigma_{q}$, on the rib acting parallel to the bar axis. Normal to the bearing stress, the minor principal stress $\sigma_{r}$ acts radially around the bar. The method of analysis (presented here is a slightly revised and condensed form) has been used previously by Choi and Lee (2002) to evaluate the bond strength in splitting.

The bond force equal to the sum of the bearing stress on a single rib area $T$, is given by

$$
T=A_{r} \sigma_{q}
$$

in which $A_{r}=$ projected area of rib parallel to the bar axis, approximated by $A_{r}=$ $\pi d_{b} h_{r}$ where $h_{r}$ is the average rib height, $\sigma_{q}=$ bearing stress on the bar rib acting parallel to the bar axis

The frictional force between the concrete and the steel on the inclined surface of the rib may be represented using the Mohr-Coulomb relation,

$$
\tau=c+\mu \sigma_{n}
$$

where $c=$ cohesion, $\mu=$ coefficient of friction, $\sigma_{n}=$ normal stress.

Suppose that the stresses along an interface with an angle of $\alpha$, defined as bearing angle, are in equilibrium with the sliding stress by $\sigma_{q}$ and the normal stress by $\sigma_{n}$. The stress $\sigma_{q}$, is given by

$$
\sigma_{q}=\left(\sigma_{r} \frac{(1+\mu \cot \alpha)}{(1-\mu \tan \alpha)}+\frac{c}{\sin \alpha(\cos \alpha-\mu \sin \alpha)}\right)
$$

Equation (3) is substituted into Eq. (1) to obtain

$$
T=A_{r}\left(\sigma_{r} \frac{(1+\mu \cot \alpha)}{(1-\mu \tan \alpha)}+\frac{c}{\sin \alpha(\cos \alpha-\mu \sin \alpha)}\right)
$$

where $\sigma_{r}$ acts radially around the bar axis on the concrete cover. The radial stress $\sigma_{r}$ acts over a distance of $d F_{x}$ below the rib, and exerts a bursting force on the concrete 
around the bar. Figure 2 shows the force, $h_{r} \cot \alpha$ exerted by $\sigma_{r}$ on one rib over a short length of the bar circumference. The component of force in the x-direction is

$$
d F_{x}=\sigma_{r} h_{r} \cot \alpha \frac{d b}{2} d \theta \cos \theta
$$

The summation of the component force on the perimeter is given by

$$
F_{X}=\int_{-\frac{\pi}{2}}^{\frac{\pi}{2}} d F_{x}=\sigma_{r} \cot \alpha h_{r} d_{b}
$$

Equation (6) is substituted in to Eq. (4), resulting in the final equation to predict bond resistance, which is expressed as follows.

$$
T_{\text {split }}=F_{x} \pi \tan \alpha \frac{(1+\mu \cot \alpha)}{(1-\mu \tan \alpha)}+\operatorname{Ar} \frac{c}{\sin \alpha(\cos \alpha-\mu \sin \alpha)}
$$

\section{Bond resistance in shearing failure}

Deformed bars bear against the concrete in front of the ribs, thus increasing shearing stress on the concrete key. Shear may cause failure, and the potential failure plane can be established for such cases along which shear stresses are high, as shown in Fig. 3. The location of shear failure surface along the possible shear crack depends on the rib geometry and the levels of vertical force (confining force) and horizontal force (bond force). Failure occurs when the shear strength of the concrete key is overcome. From the force boundary conditions, an angle $\alpha$ is made along the shear failure surface, where the tangential stresses and the radial stresses are in equilibrium. For cracks, in monolithic concrete, shear strength should not be assumed greater than $0.2 f_{c} A_{c}$ as shown in Eq. (8).

$$
V n=0.2 f^{\prime} c A c
$$

where $A_{c}$ is the area of cracked surface.

The area of cracked surface $A_{c}$ defined by the area of a cone with the angle of $\alpha$,

$$
A_{C}=\frac{\pi d_{b} h_{r}}{\sin \alpha}
$$

The concrete in contact with the bearing side of a rib is in a state of triaxial compression and is subjected to very high compression from the confining force $F_{\mathrm{x}}$. This triaxility of stress increases the shear strength of the concrete. The high compression is also beneficial to increase the shear strength, since the high compressive stress modifies the magnitude and direction of principal stress and increases the cracking load,. Two parameters accounting for the increased shear strength from the tri-axial state and the high compression, $\kappa_{1}$ and $\kappa_{2}$ are proposed Using Eq. (8) and (9) and the two parameters, the bond resistance in shearing failure is proposed by 


$$
T_{\text {shear }}=\kappa_{1} \kappa_{2} \frac{0.2 f^{\prime}{ }^{\prime} \pi d_{b} h_{r}}{\sin \alpha}
$$

where $\kappa_{1}=$ triaxial state parameter and $\kappa_{2}=$ high compression parameter.

Information on these two quantities shall be obtained from the results of future analytical or experimental studies.

\section{BEARING ANGLE MODEL}

The friction coefficient $\mu$ is one of the key variables to determine the bond resistance. Bond resistance increases as the friction coefficient increases. The contribution from cohesion to bond resistance is small and diminishes as bars slip. The confinement force $F_{X}$, provided by concrete cover or transverse reinforcement, is proportional to the bond force. The capacity of the confinement force is made up of the splitting resistance by concrete cover or by transverse reinforcement, thus the confinement force has a limitation. When the confinement is determined by the structure itself, the bearing angle is the only variable in Eq. (7) corresponding to the change of bond resistance. The bearing angle of the failure surface of the concrete in front of the ribs may be varied.

As in Eq. (10), the shearing resistance is obtained by the concrete key which would be sheared off, forming a cone with a length equal to several times the rib height. The bearing angle is, again, the key variable since the length of the cone is a function of the bearing angle. The bearing angle tends to be decreased to a smaller value, to increase the shearing bond resistance. There might be a lower limit on the bearing angle and the minimum value of the bearing angle can be obtained by the ratio of the rib spacing to the rib height.

Bond strength is determined along the interface at a state of resistance equilibrium under any failure condition. Normally, the weaker mode of the two failures, splitting and shearing failure, is considered to govern bond strength, but both failures control bond strength because two failures appears to occur simultaneously. In these cases, the bearing angle is decreased to decrease in the splitting resistance and increase in the shearing resistance. As the bearing angle reaches a certain value of the angle, then, the concrete key is sheared off. The bearing angle rotates and is determined so that the splitting resistance can be equal to the shearing resistance, and finally the resistance itself becomes bond strength $T_{\text {bond. }}$ Thus,

$$
T_{\text {split }}=T_{\text {shear }}=T_{\text {bond }}
$$

Equation (11) can be solved for the bearing angle $\alpha$. The solution for the bearing angle to determine bond strength by the bearing angle model is schematically illustrated in Fig. 4. As in cases of moderate or high confinement, when the splitting resistance is higher than the shearing resistance, the splitting 
resistance decreases with decreasing the bearing angle. As in cases of low confinement, when the shearing resistance is higher than the splitting resistance, the shearing resistance tends to be minimized and the splitting resistance tends to be maximized keeping the bearing angle as high as possible. The bearing angle rotates so that the best resistance, bond strength, is obtained from the combined effect of the splitting and shearing resistance.

\section{CONCLUSIONS}

Analytical expressions to determine the bond resistances for splitting and shearing failures are derived where the bearing angle is a key variable. As the bearing angle is decreased, the splitting bond resistance decreases while the shearing bond resistance increases. In the case of bars at a moderate level of confinement, which represents the practice, the bearing angle is decreased to decrease the splitting resistance and to increase the shearing resistance, until reaching a certain value of angle. The bearing angle rotates and is determined so that the splitting resistance can be equal to the shearing resistance and the resistance itself becomes bond strength. As bars slip further, in the case of bars confined by heavy transverse reinforcement, the bearing angle at the interface may be continually decreased by micro-crushing of the concrete key.

\section{REFERENCES}

Cairns, J., 1979, “An Analysis of the Ultimate Strength of Lapped Joints of Compression Reinforcement,” Magazine of Concrete Research, V. 31, No. 106, Mar., pp. 19-27.

Choi, O. C. and Lee, W. S., 2002, "Interfacial Bond Analysis of Deformed Bars to Concrete,” ACI Structural Journal, V. 99, No. 6, Nov.-Dec., pp. 750-756.

Lutz, L. A. and Gergely, P., 1967, "Mechanics of Bond and Slip of Deformed Bars in Concrete,” ACI Journal, Proceedings, V. 64, No. 11, Nov., pp. 711-721.

Rehm, G., 1957, “The fundamental Law of Bond,” RILEM-Symposium on Bond and Crack Formation in Reinforced Concrete, Vol. II, Stockholm, pp. 491.

Skorobogatov, S. M. and Edwards, A. D., 1979, "The influence of the Geometry of Deformed Steel Bars on Their Bond Strength in Concrete,” The Institute of Civil Engineers, Proceedings, Vol. 67, Part 2, June, 327-339.

Tepfers, R., 1979, "Cracking of Concrete Cover Along Anchored Deformed Reinforcing Bars,” Magazine of Concrete Research, V. 31, No. 106, Mar., pp. 3-12. Tholen, M. L. and Darwin, D., 1996, "Effects of Deformation Properties on the Bond of Reinforcing Bars," SM Report, No. 42, University of Kansas, page 370. 


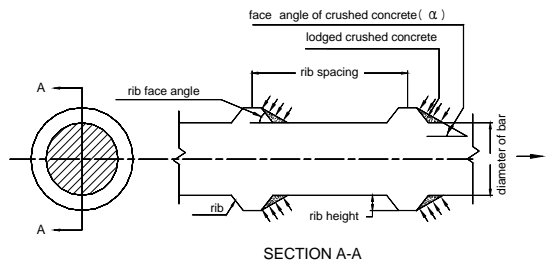

Fig. 1 Flattened rib face angleby concrete crushing (Tepfers 1979)

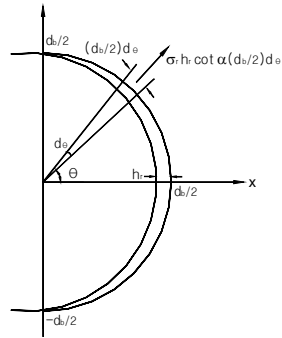

Fig.2 Radial stress around bar circumference (Cairns 1979)

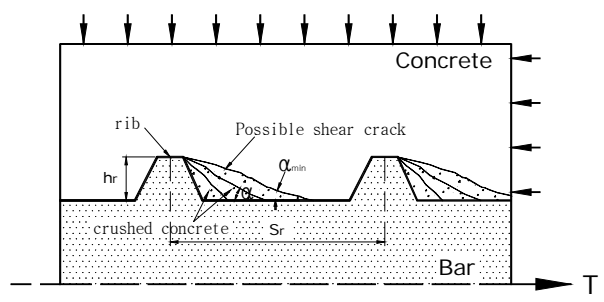

Fig. 3 Shear cracks by the concrete key between bar ribs

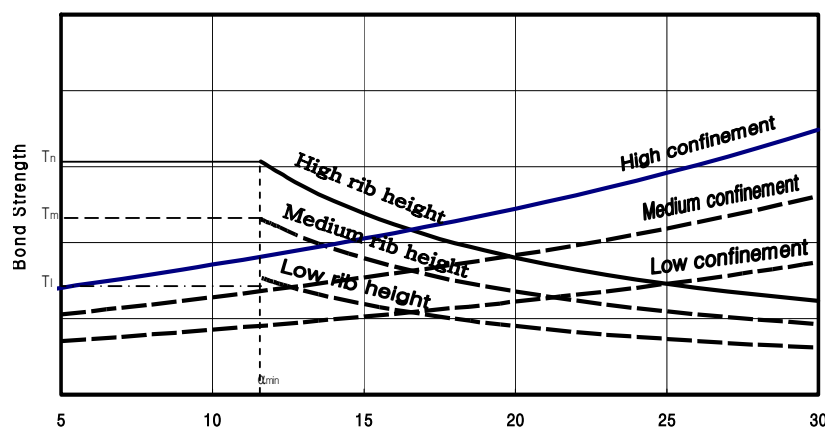

Fig. 4 Schematic for determination of bond strength by bearing angle model 